\title{
DOUBLE DIRICHLET SERIES ASSOCIATED WITH ARITHMETIC FUNCTIONS
}

\author{
KOHJI MATSUMOTO, AKIHIKO NAWASHIRO, AND HIROFUMI TSUMURA
}

\begin{abstract}
We consider double Dirichlet series associated with arithmetic functions such as the von Mangoldt function, the Möbius function, and so on. We show analytic continuations of them by use of the Mellin-Barnes integral, and determine the location of singularities. Furthermore we observe their reverse values at non-positive integer points.
\end{abstract}

\section{INTRODUCTION}

Let $\mathbb{N}$ be the set of natural numbers, $\mathbb{N}_{0}:=\mathbb{N} \cup\{0\}, \mathbb{P}$ the set of prime numbers, $\mathbb{R}$ the field of real numbers, $\mathbb{C}$ the field of complex numbers and $i:=\sqrt{-1}$.

For any arithmetic function $\alpha: \mathbb{N} \rightarrow \mathbb{C}$, let

$$
\Phi(s ; \alpha)=\sum_{n=1}^{\infty} \frac{\alpha(n)}{n^{s}}
$$

be the Dirichlet series associated with $\alpha$. It is important to show the analytic continuation of $\Phi(s ; \alpha)$ for various $\alpha$ 's. As its multiple version, we consider the multiple Dirichlet series

$$
\begin{aligned}
\Phi_{r} & \left(s_{1}, \cdots, s_{r} ; \alpha_{1}, \cdots, \alpha_{r}\right) \\
& =\sum_{m_{1}=1}^{\infty} \sum_{m_{2}=1}^{\infty} \cdots \sum_{m_{r}=1}^{\infty} \frac{\alpha_{1}\left(m_{1}\right) \alpha_{2}\left(m_{2}\right) \cdots \alpha_{r}\left(m_{r}\right)}{m_{1}^{s_{1}}\left(m_{1}+m_{2}\right)^{s_{2}} \cdots\left(m_{1}+\cdots+m_{r}\right)^{s_{r}}},
\end{aligned}
$$

where $\alpha_{k}(1 \leq k \leq r)$ are arithmetic functions. The typical example of (1.2) is the multiple zeta function of Euler-Zagier type defined by

$$
\zeta_{r}\left(s_{1}, \cdots, s_{r}\right)=\sum_{m_{1}=1}^{\infty} \sum_{m_{2}=1}^{\infty} \cdots \sum_{m_{r}=1}^{\infty} m_{1}^{-s_{1}}\left(m_{1}+m_{2}\right)^{-s_{2}} \cdots\left(m_{1}+\cdots+m_{r}\right)^{-s_{r}},
$$

which has been studied extensively in these two decades. In fact, the meromorphic continuation of (1.3) to the whole space $\mathbb{C}^{r}$ has been proved by Akiyama, Egami and Tanigawa [1, Zhao [24], the first-named author [14, 15], and so on. In particular, the method in [14, 15] is to make use of the Mellin-Barnes integral formula (see, for example, [23, Section 14.51, p.289, Corollary]):

$$
(1+\lambda)^{-s}=\frac{1}{2 \pi i} \int_{(c)} \frac{\Gamma(s+z) \Gamma(-z)}{\Gamma(s)} \lambda^{z} d z
$$

2000 Mathematics Subject Classification. 11M41, 11M06, 11M26.

Key words and phrases. Multiple Dirichlet series; Riemann zeta function; von Mangoldt function; Möbius function.

This work was supported by Japan Society for the Promotion of Science, Grant-in-Aid for Scientific Research No. 25287002 (K. Matsumoto) and No. 15K04788 (H. Tsumura). 
where $s, \lambda \in \mathbb{C}$ with $\lambda \neq 0,|\arg \lambda|<\pi, \Re s>0, c \in \mathbb{R}$ with $-\Re s<c<0$, and the path of integration is the vertical line form $c-i \infty$ to $c+i \infty$. This method is based on Katsurada's results [10, 11.

By applying this method, Tanigawa and the first-named author proved the following general result. (We also mention a paper of de la Bretèche [3] who treated similar multiple series.) Let $\mathcal{A}$ be the set of arithmetic functions satisfying the following three conditions: If $\alpha \in \mathcal{A}$, then

(I) $\Phi(s ; \alpha)$ is absolutely convergent for $\Re s>\delta=\delta(\alpha)(>0)$;

(II) $\Phi(s ; \alpha)$ can be continued meromorphically to the whole plane $\mathbb{C}$, holomorphic except for a possible pole (of order at most 1) at $s=\delta$;

(III) in any fixed strip $\sigma_{1} \leq \sigma \leq \sigma_{2}, \Phi(\sigma+i t ; \alpha)=O\left(|t|^{A}\right)$ holds as $|t| \rightarrow \infty$, where $A$ is a non-negative constant.

Theorem 1.1 (Matsumoto-Tanigawa[17, Theorem 1]). If arithmetic functions $\alpha_{1}, \ldots, \alpha_{r}$ belong to $\mathcal{A}$, then $\Phi_{r}\left(s_{1}, \cdots, s_{r} ; \alpha_{1}, \cdots, \alpha_{r}\right)$ can be continued meromorphically to the whole space $\mathbb{C}^{r}$, and location of its possible singularities can be described explicitly. In particular, if all $\Phi\left(s ; \alpha_{k}\right)$ 's are entire, then $\Phi_{r}\left(s_{1}, \cdots, s_{r} ; \alpha_{1}, \cdots, \alpha_{r}\right)$ is also entire.

They applied this result to multiple Dirichlet $L$-functions of Euler-Zagier type and multiple automorphic $L$-functions.

On the other hand, as an example which is outside of Theorem 1.1. Egami and the first-named author [5] considered the double series associated with the von Mangoldt function. Let $\zeta(s)$ be the Riemann zeta function and denote by $\left\{\rho_{n}\right\}_{n \geq 1}$ the non-trivial zeros of $\zeta(s)$ numbered by the size of absolute values of their imaginary parts. Let $\Lambda$ be the von Mangoldt function defined by

$$
\Lambda(n)=\left\{\begin{array}{ll}
\log p & \left(n=p^{m} \text { for } p \in \mathbb{P}, m \in \mathbb{N}\right) \\
0 & \text { (otherwise) }
\end{array} .\right.
$$

Then it is well-known that

$$
\Phi(s ; \Lambda)=\sum_{n=1}^{\infty} \frac{\Lambda(n)}{n^{s}}=-\frac{\zeta^{\prime}(s)}{\zeta(s)}
$$

(see [22, $\S 1.1]$ ). We denote this function by $M(s)$. We see that $M(s)$ has poles at $s=1, s=-2 m(m \in \mathbb{N})$ and $s=\rho_{l}(l \in \mathbb{N})$, hence does not satisfy the assumption (II). That is, $\Lambda \notin \mathcal{A}$. As a double version of $M(s)$, they considered

$$
\mathcal{M}_{2}(s)=\sum_{m_{1}=1}^{\infty} \sum_{m_{2}=1}^{\infty} \frac{\Lambda\left(m_{1}\right) \Lambda\left(m_{2}\right)}{\left(m_{1}+m_{2}\right)^{s}}\left(=\Phi_{2}(0, s ; \Lambda, \Lambda)\right)
$$

which can be written as $\sum_{n=1}^{\infty} G_{2}(n) n^{-s}$, where

$$
G_{2}(n)=\sum_{m_{1}+m_{2}=n} \Lambda\left(m_{1}\right) \Lambda\left(m_{2}\right)
$$

It should be emphasized that $\mathcal{M}_{2}(s)$ is closely connected to the famous Goldbach conjecture which implies that $G_{2}(n)>0$ for all even $n \geq 4$. The study of $\mathcal{M}_{2}(s)$ will be useful to understand the behaviour of $G_{2}(n)$. However, we cannot apply Theorem 1.1 to $\mathcal{M}_{2}(s)=\Phi_{2}(0, s ; \Lambda, \Lambda)$.

They also showed that the line $\Re s=1$ is the natural boundary of $\mathcal{M}_{2}(s)$ under some plausible assumptions, hence $\mathcal{M}_{2}(s)$ cannot be continued meromorphically to the whole complex plane $\mathbb{C}$ (see [5, Theorem 2.2]). 

by

In the present paper we first consider another type of double version of $M(s)$ defined

$$
\Phi_{2}\left(s_{1}, s_{2} ; 1, \Lambda\right)=\sum_{m_{1}=1}^{\infty} \sum_{m_{2}=1}^{\infty} \frac{\Lambda\left(m_{2}\right)}{m_{1}^{s_{1}}\left(m_{1}+m_{2}\right)^{s_{2}}} .
$$

The right-hand side of (1.5) is absolutely convergent for $\Re s_{2}>1, \Re\left(s_{1}+s_{2}\right)>2$. However, similar to $\mathcal{M}_{2}(s)$, we cannot apply Theorem 1.1 to $\Phi_{2}\left(s_{1}, s_{2} ; 1, \Lambda\right)$. The first main aim of this paper is to prove that $\Phi_{2}\left(s_{1}, s_{2} ; 1, \Lambda\right)$ can be continued meromorphically to the whole space $\mathbb{C}^{2}$ (see Theorem 2.1), and location of its singularities can be described explicitly (see Theorem 2.2),

We here remark that

$$
\Phi_{2}(0, s ; 1, \Lambda)=\sum_{n=1}^{\infty} \frac{\psi(n-1)}{n^{s}},
$$

where $\psi(x)$ is the Chebyshev $\psi$-function defined by $\psi(x)=\sum_{1 \leq n \leq x} \Lambda(n)$ (see [21, Chap. $7, \S 2]$ ). It is well-known that the prime number theorem comes from $\psi(x) \sim x$ $(x \rightarrow \infty)$, and also from $\widehat{\psi}(x) \sim \frac{1}{2} x^{2}(x \rightarrow \infty)$, where

$$
\widehat{\psi}(x)=\sum_{2 \leq n \leq x} \psi(n-1)\left(=\int_{1}^{x-1} \psi(u) d u\right)
$$

(see [21, Chap. 7, Propositions 2.1 and 2.2]). Note that applying Perron's formula to (1.6), we have

$$
\widehat{\psi}(x)=\frac{1}{2 \pi i} \int_{c-i T}^{c+i T} \Phi_{2}(0, s ; 1, \Lambda) \frac{x^{s}}{s} d s+(\text { error term }) \quad(c>2) .
$$

From this viewpoint as well, it seems important to study $\Phi_{2}\left(s_{1}, s_{2} ; 1, \Lambda\right)$.

Secondly, for any $\Phi(s ; \alpha)$ with $\alpha \in \mathcal{A}$, we define $\widetilde{\alpha}: \mathbb{N} \rightarrow \mathbb{C}$ by

$$
\frac{\Phi(s ; \alpha)}{\zeta(s)}=\sum_{n=1}^{\infty} \frac{\widetilde{\alpha}(n)}{n^{s}}(=: \Phi(s ; \widetilde{\alpha})),
$$

and consider the double series

$$
\Phi_{2}\left(s_{1}, s_{2} ; 1, \widetilde{\alpha}\right)=\sum_{m_{1}=1}^{\infty} \sum_{m_{2}=1}^{\infty} \frac{\widetilde{\alpha}\left(m_{2}\right)}{m_{1}^{s_{1}}\left(m_{1}+m_{2}\right)^{s_{2}}} .
$$

The second main aim of this paper is to prove that $\Phi_{2}\left(s_{1}, s_{2} ; 1, \widetilde{\alpha}\right)$ can be continued meromorphically to the whole space $\mathbb{C}^{2}$ (see Theorem 4.3). Note that we can apply this result to the cases when $\widetilde{\alpha}$ is the Möbius function $\mu$, the Euler totient function $\phi$, and so on (see Section (6). We further calculate reverse values of $\Phi_{2}\left(s_{1}, s_{2} ; 1, \Lambda\right)$ at points on the sets of singularity (see Propositions 5.1 and 5.4 and Example [5.3), and also those of $\Phi_{2}\left(s_{1}, s_{2} ; 1, \mu\right)$ (see Example 6.1).

If $\widetilde{\alpha}$ is an arithmetic function for which $\Phi(s ; \widetilde{\alpha})$ has only finitely many poles, then the double series of the form (1.8) has been studied by Choie and the first-named author [4]. However $\Phi(s ; \widetilde{\alpha})$ defined by (1.7) obviously has infinitely many poles, so is outside of the study in [4.

Also, since $\zeta^{\prime}(s)$ has a double pole at $s=1$, it does not satisfy the assumption (II). Therefore we will consider the case of the von Mangoldt function separately in Section 2. The other reason of this separate treatment is that we need not assume Assumption 4.1 in Section 2,

The authors believe that it is not difficult to extend the results in the present paper to the case when $\Phi(s ; \widetilde{\alpha})$ has a pole of higher order at $s=\delta$, or even to the case when $\Phi(s ; \widetilde{\alpha})$ has other finitely many poles. 
2. The DOUble SERIES $\Phi_{2}\left(s_{1}, s_{2} ; 1, \Lambda\right)$

In this section, we consider analytic properties of $\Phi_{2}\left(s_{1}, s_{2} ; 1, \Lambda\right)$. We first define $a_{k}$ and $b_{l}$ by

$$
\begin{array}{ll}
a_{k}=\frac{1}{2 \pi i} \int_{|\xi+k|=\frac{1}{2}} \frac{M(\xi)}{\xi+k} d \xi & (k \in \mathbb{N} ; k: \text { even }), \\
b_{l}=\frac{1}{2 \pi i} \int_{|\xi+l|=\frac{1}{2}} \frac{\Gamma(\xi)}{\xi+l} d \xi & \left(l \in \mathbb{N}_{0}\right),
\end{array}
$$

namely $a_{k}$ and $b_{l}$ are constant terms of Laurent series of $M(s)=-\zeta^{\prime}(s) / \zeta(s)$ at $s=-k$ and of $\Gamma(s)$ at $s=-l$, respectively. We can also express that

$$
\begin{aligned}
a_{k} & =\lim _{s \rightarrow-k} \frac{d}{d s}(s+k) M(s)=-\frac{\zeta^{\prime \prime}(-k)}{2 \zeta^{\prime}(-k)} \quad(k \text { :even }), \\
b_{l} & =\lim _{s \rightarrow-l} \frac{d}{d s}(s+l) \Gamma(s)=\frac{(-1)^{l}}{l !}\left(\sum_{j=1}^{l} \frac{1}{j}-\gamma\right),
\end{aligned}
$$

where $\gamma$ is the Euler constant.

Theorem 2.1. $\Phi_{2}\left(s_{1}, s_{2} ; 1, \Lambda\right)$ can be continued meromorphically to the whole space $\mathbb{C}^{2}$ by the following expression:

$$
\begin{aligned}
& \Phi_{2}\left(s_{1}, s_{2} ; 1, \Lambda\right) \\
& =\frac{\zeta\left(s_{1}+s_{2}-1\right)}{s_{2}-1}-(\log 2 \pi) \zeta\left(s_{1}+s_{2}\right) \\
& +\sum_{\substack{k=1 \\
k=\text { odd }}}^{N-1}\left(\begin{array}{c}
-s_{2} \\
k
\end{array}\right) M(-k) \zeta\left(s_{1}+s_{2}+k\right) \\
& -\sum_{\substack{k=1 \\
k: \text { even }}}^{N-1}\left[\left(\begin{array}{c}
-s_{2} \\
k
\end{array}\right)\left\{\left(-a_{k}+k ! b_{k}\right) \zeta\left(s_{1}+s_{2}+k\right)-\zeta^{\prime}\left(s_{1}+s_{2}+k\right)\right\}\right. \\
& \left.\quad-\frac{1}{k !} \frac{\Gamma^{\prime}\left(s_{2}+k\right)}{\Gamma\left(s_{2}\right)} \zeta\left(s_{1}+s_{2}+k\right)\right] \\
& -\frac{1}{\Gamma\left(s_{2}\right)} \sum_{n=1}^{\infty} \operatorname{ord}\left(\rho_{n}\right) \Gamma\left(s_{2}-\rho_{n}\right) \Gamma\left(\rho_{n}\right) \zeta\left(s_{1}+s_{2}-\rho_{n}\right) \\
& +\frac{1}{2 \pi i \Gamma\left(s_{2}\right)} \int_{(N-\varepsilon)} \Gamma\left(s_{2}+z\right) \Gamma(-z) M(-z) \zeta\left(s_{1}+s_{2}+z\right) d z,
\end{aligned}
$$

where $N \in \mathbb{N}$, $\varepsilon$ is a small positive number, and $\operatorname{ord}\left(\rho_{n}\right)$ is the order of $\rho_{n}$ as a zero of the Riemann zeta function.

Proof. We first assume $\Re s_{2}>1, \Re\left(s_{1}+s_{2}\right)>2$ and $\max \left\{-\Re s_{2}, 1-\Re\left(s_{1}+s_{2}\right)\right\}<c<$ -1 . Then we have

$$
\begin{aligned}
\Phi_{2}\left(s_{1}, s_{2} ; 1, \Lambda\right) & =\sum_{m=1}^{\infty} \sum_{n=1}^{\infty} \frac{\Lambda(n)}{m^{s_{1}}(m+n)^{s_{2}}} \\
& =\sum_{m=1}^{\infty} \sum_{n=1}^{\infty} \frac{\Lambda(n)}{m^{s_{1}+s_{2}}}\left(1+\frac{n}{m}\right)^{-s_{2}} .
\end{aligned}
$$


We apply the Mellin-Barnes formula (1.4) with $s=s_{2}, \lambda=n / m$ to 2.4 to obtain

$$
\begin{aligned}
\Phi_{2}\left(s_{1}, s_{2} ; 1, \Lambda\right) & =\sum_{m=1}^{\infty} \sum_{n=1}^{\infty} \frac{\Lambda(n)}{m^{s_{1}+s_{2}}} \frac{1}{2 \pi i} \int_{(c)} \frac{\Gamma\left(s_{2}+z\right) \Gamma(-z)}{\Gamma\left(s_{2}\right)}\left(\frac{n}{m}\right)^{z} d z \\
= & \frac{1}{2 \pi i} \int_{(c)} \frac{\Gamma\left(s_{2}+z\right) \Gamma(-z)}{\Gamma\left(s_{2}\right)} \sum_{n=1}^{\infty} \Lambda(n) n^{z} \sum_{m=1}^{\infty} m^{-s_{1}-s_{2}-z} d z \\
= & \frac{1}{2 \pi i} \int_{(c)} \frac{\Gamma\left(s_{2}+z\right) \Gamma(-z)}{\Gamma\left(s_{2}\right)} M(-z) \zeta\left(s_{1}+s_{2}+z\right) d z .
\end{aligned}
$$

The exchange between the summation and the integration is valid because of the choice of $c$.

Now, by the same argument as in [5, Section 3], we shift the path $(c)$ to $(N-\varepsilon)$ for an arbitrarily large $N>0$. In order to check the validity of this shifting, we prepare the known order estimations of $\Gamma(s), \zeta(s)$ and $M(s)$ as follows. First, we recall that

$$
\begin{gathered}
\Gamma(\sigma+i t)=O\left(|t|^{\sigma-\frac{1}{2}} e^{-\frac{\pi}{2}|t|}\right) \\
\zeta(\sigma+i t)=O\left(|t|^{A}\right) \quad(A \text { : positive constant })
\end{gathered}
$$

hold uniformly in fixed vertical strips (see [23, § 12.3] and [22, p.95 (5.1.1)]). Next we can find an arbitrarily large $t_{0}>0$ such that

$$
\left|t_{0}-\Im\left(\rho_{n}\right)\right| \gg\left(\log t_{0}\right)^{-1}
$$

for any $n \in \mathbb{N}$ (see [5, (3.5)]). Then it holds that

$$
M\left(\sigma+i t_{0}\right) \ll\left(\log t_{0}\right)^{2} \quad(-1 \leq \sigma \leq 2)
$$

(see [5, (3.7)] or Ingham [8, Theorem 26]). Furthermore it holds that

$$
M(\sigma+i t) \ll \log (2|\sigma+i t|) \quad(\sigma<-1, t>0),
$$

provided that discs of radii $1 / 2$ around the trivial zeros $s=-2 m(m \in \mathbb{N})$ of $\zeta(s)$ are excluded (see Ivić 9, (12.22)] or [8, Theorem 27]). Using these results, we see that the above shifting is possible by the same argument as in [5, Section 3].

In the course of this shifting, we encounter the poles of the integrand which are derived from $\Gamma(-z)$ and $M(-z)$. All poles of $\Gamma(-z)$ and $M(-z)$ are simple and the residues at those poles are

$$
\begin{gathered}
\operatorname{Res}_{z=-1} M(-z)=-1, \\
\operatorname{Res}_{z=-\rho_{n}} M(-z)=\operatorname{ord}\left(\rho_{n}\right), \\
\operatorname{Res}_{z=k} M(-z)=1 \quad(1 \leq k \leq N-1, k: \text { even })
\end{gathered}
$$

and

$$
\operatorname{Res}_{z=k} \Gamma(-z)=\frac{(-1)^{k-1}}{k !} \quad(0 \leq k \leq N-1) .
$$

Therefore the integrand has simple poles at $z=-1, z=0, z=\rho_{n}(n \in \mathbb{N}), z=k$ $(1 \leq k \leq N-1, k$ : odd $)$, and has double poles at $z=k(2 \leq k \leq N-1, k$ : even) whose residues are

$$
\begin{aligned}
& \frac{1}{\Gamma\left(s_{2}\right)}\left\{\Gamma\left(s_{2}+k\right)\left(\frac{(-1)^{k-1}}{k !} a_{k}+b_{k}\right) \zeta\left(s_{1}+s_{2}+k\right)\right. \\
& \left.\quad+\frac{(-1)^{k-1}}{k !}\left(\Gamma\left(s_{2}+k\right) \zeta^{\prime}\left(s_{1}+s_{2}+k\right)+\Gamma^{\prime}\left(s_{2}+k\right) \zeta\left(s_{1}+s_{2}+k\right)\right)\right\} \\
& =\left(\begin{array}{c}
-s_{2} \\
k
\end{array}\right)\left\{\left(-a_{k}+k ! b_{k}\right) \zeta\left(s_{1}+s_{2}+k\right)-\zeta^{\prime}\left(s_{1}+s_{2}+k\right)\right\}
\end{aligned}
$$




$$
-\frac{1}{k !} \frac{\Gamma^{\prime}\left(s_{2}+k\right)}{\Gamma\left(s_{2}\right)} \zeta\left(s_{1}+s_{2}+k\right)
$$

hence we obtain (2.3). The first, the second, the third and the fourth terms on the right-hand side of (2.3) are obviously meromorphic on the whole space $\mathbb{C}^{2}$.

The fifth term is convergent absolutely for all $s_{1}, s_{2} \in \mathbb{C}$ except for its singularities $s_{1}+s_{2}=1+\rho_{n}$ and $s_{2}=\rho_{n}-j\left(j \in \mathbb{N}_{0}\right)$ because of the order estimations (2.6), 2.7) and

$$
\operatorname{ord}\left(\rho_{n}\right)=O\left(\log \left|\rho_{n}\right|\right),
$$

which can be easily obtained from

$$
N(T+1)-N(T)=O(\log T),
$$

where $N(T)$ is the number of non-trivial zeros (counted with multiplicity) of $\zeta(\sigma+i t)$ in the region $-T \leq t \leq T$ (see [22, Chapter 9, p.214 (9.4.3)]).

The integral on the right-hand side of (2.3) can be analytically continued to the region

$$
\mathcal{D}_{N}=\left\{\left(s_{1}, s_{2}\right) \in \mathbb{C}^{2} \mid \Re s_{2}>-N+\varepsilon, \Re\left(s_{1}+s_{2}\right)>1-N+\varepsilon\right\},
$$

because in this region the poles of the integrand are not on the path of integration. Since $N$ is arbitrary, (2.3) gives the analytic continuation of $\Phi_{2}\left(s_{1}, s_{2} ; 1, \Lambda\right)$ to the whole space $\mathbb{C}^{2}$. Thus we complete the proof of Theorem 2.1.

From Theorem 2.1, we can prove the following.

Theorem 2.2. The singularities of $\Phi_{2}\left(s_{1}, s_{2} ; 1, \Lambda\right)$ are located only on the subsets of $\mathbb{C}^{2}$ defined by one of the following equations:

$$
\begin{aligned}
& s_{2}=1, \\
& s_{2}=-l \quad(l \in \mathbb{N}, \quad l \geq 2), \\
& s_{1}+s_{2}=2-l \quad\left(l \in \mathbb{N}_{0}\right), \\
& s_{2}=-l+\rho_{n} \quad\left(n \in \mathbb{N}, l \in \mathbb{N}_{0}\right), \\
& s_{1}+s_{2}=1+\rho_{n} \quad(n \in \mathbb{N}),
\end{aligned}
$$

all of which are "true" singularities.

Proof. From (2.3) it is easy to see that possible singularities of $\Phi_{2}\left(s_{1}, s_{2} ; 1, \Lambda\right)$ are located only on the hyperplanes defined by the above list.

We prove that all of those are true singularities. Fix an arbitrary $N \in \mathbb{N}$ and consider (2.3) on $\mathcal{D}_{N}$ defined by (2.11). We put $s_{1}+s_{2}=u$, and regard (2.3) as a formula in variables $u, s_{2}$. This idea of "changing variables" is originally due to Akiyama, Egami and Tanigawa [1]. For simplicity, we write the right-hand side of (2.3) as

$$
X_{1}-X_{2}+\sum_{\substack{k=1 \\ k: \text { odd }}}^{N-1} Y_{1, k}-\sum_{\substack{k=1 \\ k: \text { even }}}^{N-1} Y_{2, k}-\sum_{n=1}^{\infty} Z_{n}+\text { (integral part). }
$$

We can see that the integral part is absolutely convergent for any $u, s_{2} \in \mathcal{D}_{N}$, namely is holomorphic. We have possible singularities $u=2$ and $s_{2}=1$ arising from $X_{1}, u=1$ from $X_{2}, u=1-k$ ( $k$ : odd) from $Y_{1, k}$.

Next consider $Y_{2, k}$. The possible singularities coming from this term are $u=1-k$ ( $k:$ even) and $s_{2}=-k-m$ ( $k:$ even, $m \in \mathbb{N}_{0}$ ). The Laurent series of $Y_{2, k}$ around $u=1-k$ has the principal part of order 2 because of the term $\zeta^{\prime}(u+k)$, hence this is indeed a candidate of singularity. Also, since

$$
\frac{\Gamma^{\prime}(s+k)}{\Gamma(s)}=\frac{(-1)^{k-1}(k+m) !}{m !} \frac{1}{s+k+m}+O(1) \quad(s \rightarrow-k-m)
$$


for even $k \geq 2$ and $m \in \mathbb{N}_{0}$, the principal part of the Laurent series of $Y_{2, k}$ around $s_{2}=-l(l \in \mathbb{N}, l \geq 2)$ is

$$
\sum_{\substack{k+m=l \\
k: \text { even }}} \frac{\zeta(u+k)}{k !} \frac{(-1)^{k-1} l !}{m !} \frac{1}{s_{2}+l}=\sum_{\substack{k=2 \\
k: \text { even }}}^{l}(-1)^{k-1}\left(\begin{array}{l}
l \\
k
\end{array}\right) \zeta(u+k) \frac{1}{s_{2}+l},
$$

whose coefficient does not vanish identically as a function in $u$. In fact, when $l$ is even, by setting $u=-l$, the coefficient equals to

$$
\sum_{\substack{k=2 \\
k: \text { even }}}^{l}(-1)^{k-1}\left(\begin{array}{l}
l \\
k
\end{array}\right) \zeta(-l+k)=(-1)^{l-1} \zeta(0) \neq 0
$$

because negative even integers are trivial zeros of the Riemann zeta-function. When $l$ is odd, by setting $u=-l+1$, the coefficient equals to

$$
(-1)^{l}\left(\begin{array}{c}
l \\
l-1
\end{array}\right) \zeta(0) \neq 0
$$

Therefore $s_{2}=-l(l \in \mathbb{N}, l \geq 2)$ are candidates of singularities. From $Z_{n}$, we have possible singularities $s_{2}=-l+\rho_{n}(l \in \mathbb{N}, l \geq 2)$. Consequently we obtain the list (2.12)-(2.16), and all of which cannot be cancelled each other. Therefore all of them are true singularities.

Remark 2.3. Akiyama, Egami and Tanigawa observed that sets of singularities of $\zeta_{r}\left(s_{1}, \ldots, s_{r}\right)$ include many points of indeterminacy, like $\left(s_{1}, s_{2}\right)=(0,0)$ of the function $s_{1} /\left(s_{1}+s_{2}\right)$ (see [1, Section 3]). At those points, the values of $\zeta_{r}\left(s_{1}, \ldots, s_{r}\right)$ can be determined only as a limit value, depending on a choice of limiting process. As for $\Phi_{2}\left(s_{1}, s_{2} ; 1, \Lambda\right)$, we can see that the similar situation occurs from (2.3). In later sections, we will observe this type of special values.

\section{The DOUBle SERIES $\Phi_{2}\left(s_{1}, s_{2} ; 1, \widetilde{\alpha}\right)$}

In this section, by the same principle as in the previous section, we consider a general class of double series $\Phi_{2}\left(s_{1}, s_{2} ; 1, \widetilde{\alpha}\right)$ defined by (1.8).

It is well-known that

$$
\frac{1}{\zeta(s)}=\sum_{n=1}^{\infty} \frac{\mu(n)}{n^{s}} \quad(\Re s>1)
$$

where $\mu$ is the Möbius function defined for $n \in \mathbb{N}$ by

$$
\mu(n)= \begin{cases}1 & (n=1), \\ (-1)^{r} & \left(n: \text { squarefree and } n=p_{1} \cdots p_{r}, p_{i} \in \mathbb{P}\right), \\ 0 & (n: \text { not squarefree })\end{cases}
$$

(see [22, $\S 1.1]$ ). Hence, for $\Re s>\max \{1, \delta\}$, we have

$$
\frac{\Phi(s ; \alpha)}{\zeta(s)}=\sum_{m=1}^{\infty} \sum_{n=1}^{\infty} \frac{\alpha(m) \mu(n)}{(m n)^{s}} .
$$

Therefore (1.7) implies

$$
\widetilde{\alpha}(n)=\sum_{\substack{1 \leq d \leq n \\ d \mid n}} \alpha\left(\frac{n}{d}\right) \mu(d) .
$$

Proposition 3.1. If $\alpha$ satisfies the condition (I) of the class $\mathcal{A}$, we see that $\Phi_{2}\left(s_{1}, s_{2} ; 1, \widetilde{\alpha}\right)$ is absolutely convergent in the region

$$
\left\{\left(s_{1}, s_{2}\right) \in \mathbb{C}^{2} \mid \Re s_{2}>\max \{1, \delta\}, \Re\left(s_{1}+s_{2}\right)>\max \{2,1+\delta\}\right\} .
$$


Proof. Set $\sigma_{j}=\Re s_{j}$ for $j=1,2$. By (3.2), we have

$$
|\widetilde{\alpha}(n)| \leq \sum_{\substack{1 \leq d \leq n \\ d \mid n}}|\alpha(d)|
$$

Hence we have

$$
\begin{aligned}
& \sum_{m=1}^{\infty} \sum_{n=1}^{\infty}\left|\frac{\widetilde{\alpha}(n)}{m^{s_{1}}(m+n)^{s_{2}}}\right| \leq \sum_{m=1}^{\infty} \sum_{n=1}^{\infty} \frac{\sum_{d \mid n}|\alpha(d)|}{m^{\sigma_{1}}(m+n)^{\sigma_{2}}} \\
& \quad=\sum_{d=1}^{\infty}|\alpha(d)| \sum_{m=1}^{\infty} \sum_{l=1}^{\infty} \frac{1}{m^{\sigma_{1}}(m+d l)^{\sigma_{2}}} \\
& \quad \leq \sum_{d=1}^{\infty}|\alpha(d)|\left(\sum_{\substack{m, l \geq 1 \\
m \leq d l}} \frac{1}{m^{\sigma_{1}}(d l)^{\sigma_{2}}}+\sum_{\substack{m, l \geq 1 \\
m>d l}} \frac{1}{m^{\sigma_{1}+\sigma_{2}}}\right) \\
& \quad=\sum_{d=1}^{\infty} \frac{|\alpha(d)|}{d^{\sigma_{2}}} \sum_{l=1}^{\infty} \frac{1}{l^{\sigma_{2}}} \sum_{1 \leq m \leq d l} \frac{1}{m^{\sigma_{1}}}+\sum_{d=1}^{\infty}|\alpha(d)| \sum_{l=1}^{\infty} \sum_{m>d l} \frac{1}{m^{\sigma_{1}+\sigma_{2}}} \\
& \quad=: \Sigma_{1}+\Sigma_{2},
\end{aligned}
$$

say. As for $\Sigma_{2}$, we first assume $\sigma_{1}+\sigma_{2}>1$. Then we have

$$
\sum_{m>d l} \frac{1}{m^{\sigma_{1}+\sigma_{2}}} \leq \int_{d l}^{\infty} \frac{d t}{t^{\sigma_{1}+\sigma_{2}}}=\left[\frac{t^{1-\sigma_{1}-\sigma_{2}}}{1-\sigma_{1}-\sigma_{2}}\right]_{d l}^{\infty} \ll(d l)^{1-\sigma_{1}-\sigma_{2}} .
$$

Hence we obtain

$$
\begin{aligned}
\Sigma_{2} & \ll \sum_{d=1}^{\infty}|\alpha(d)| \sum_{l=1}^{\infty}(d l)^{1-\sigma_{1}-\sigma_{2}} \\
& =\sum_{d=1}^{\infty} \frac{|\alpha(d)|}{d^{\sigma_{1}+\sigma_{2}-1}} \sum_{l=1}^{\infty} \frac{1}{l^{\sigma_{1}+\sigma_{2}-1}} .
\end{aligned}
$$

By the condition (I) of the class $\mathcal{A}$, we see that the first and the second sums are convergent for $\sigma_{1}+\sigma_{2}-1>\delta$ and $\sigma_{1}+\sigma_{2}-1>1$, respectively. Therefore $\Sigma_{2}$ is convergent for

$$
\sigma_{1}+\sigma_{2}>\max \{2,1+\delta\}
$$

As for $\Sigma_{1}$, we remark that

$$
\sum_{1 \leq m \leq d l} \frac{1}{m^{\sigma_{1}}} \ll \begin{cases}1 & \left(\sigma_{1}>1\right) \\ \log (d l) & \left(\sigma_{1}=1\right) \\ (d l)^{1-\sigma_{1}} & \left(\sigma_{1}<1\right) .\end{cases}
$$

If $\sigma_{1}>1$, we have

$$
\Sigma_{1} \ll \sum_{d=1}^{\infty} \frac{|\alpha(d)|}{d^{\sigma_{2}}} \sum_{l=1}^{\infty} \frac{1}{l^{\sigma_{2}}},
$$

where the first and the second sums are convergent for $\sigma_{2}>\delta$ and $\sigma_{2}>1$, respectively. Hence $\Sigma_{1}$ in this case is convergent for $\sigma_{2}>\max \{1, \delta\}$.

If $\sigma_{1}=1$, we have

$$
\begin{aligned}
\Sigma_{1} & \ll \sum_{d=1}^{\infty} \frac{|\alpha(d)|}{d^{\sigma_{2}}} \sum_{l=1}^{\infty} \frac{\log (d l)}{l^{\sigma_{2}}} \\
& \ll \sum_{d=1}^{\infty} \frac{|\alpha(d)| \log d}{d^{\sigma_{2}}} \sum_{l=1}^{\infty} \frac{1}{l^{\sigma_{2}}}+\sum_{d=1}^{\infty} \frac{|\alpha(d)|}{d^{\sigma_{2}}} \sum_{l=1}^{\infty} \frac{\log l}{l^{\sigma_{2}}} .
\end{aligned}
$$


As well as the case $\sigma_{1}>1$, we see that $\Sigma_{1}$ in this case is convergent for $\sigma_{2}>\max \{1, \delta\}$. If $\sigma_{1}<1$, we have

$$
\Sigma_{1} \ll \sum_{d=1}^{\infty} \frac{|\alpha(d)|}{d^{\sigma_{2}}} \sum_{l=1}^{\infty} \frac{(d l)^{1-\sigma_{1}}}{l^{\sigma_{2}}}=\sum_{d=1}^{\infty} \frac{|\alpha(d)|}{d^{\sigma_{1}+\sigma_{2}-1}} \sum_{l=1}^{\infty} \frac{1}{l^{\sigma_{1}+\sigma_{2}-1}} .
$$

Hence we can similarly see that $\Sigma_{1}$ in this case is convergent for $\sigma_{1}+\sigma_{2}>\max \{2,1+\delta\}$. Combining these three cases, we see that $\Sigma_{1}$ is convergent for

$$
\sigma_{1} \geq 1, \sigma_{2}>\max \{1, \delta\}
$$

or

$$
\sigma_{1}<1, \sigma_{1}+\sigma_{2}>\max \{2,1+\delta\}
$$

We obviously see that the region (3.4) includes both the regions (3.5) and (3.6). Hence, denoting by $\Omega$ the union of regions (3.5) and (3.6), we see that $\Phi_{2}\left(s_{1}, s_{2} ; 1, \widetilde{\alpha}\right)$ is absolutely convergent in $\Omega$. We remark that

$$
\Omega= \begin{cases}\left\{\Re s_{1} \geq 1, \Re s_{2}>1\right\} \cup\left\{\Re s_{1}<1, \Re s_{1}+\Re s_{2}>2\right\} & (\delta<1), \\ \left\{\Re s_{1} \geq 1, \Re s_{2}>\delta\right\} \cup\left\{\Re s_{1}<1, \Re s_{1}+\Re s_{2}>1+\delta\right\} & (\delta \geq 1) .\end{cases}
$$

Therefore we see that $\Omega$ is equal to the region (3.3). This completes the proof.

Remark 3.2. It is known that $\zeta_{2}\left(s_{1}, s_{2}\right)$ is absolutely convergent in the region $\Re s_{2}>$ $1, \Re\left(s_{1}+s_{2}\right)>2$ (see [13, (3.2)]). Proposition 3.1 gives a generalization of this fact.

\section{Analytic Continuation of $\Phi_{2}\left(s_{1}, s_{2} ; 1, \widetilde{\alpha}\right)$}

In this section, we assume $\alpha \in \mathcal{A}$, Moreover we assume the following condition for $\zeta(s)$.

Assumption 4.1. Assume that all non-trivial zeros of $\zeta(s)$ are simple and that

$$
\frac{1}{\zeta^{\prime}\left(\rho_{n}\right)}=O\left(\left|\rho_{n}\right|^{B}\right) \quad(n \rightarrow \infty)
$$

with some constant $B>0$.

Note that (4.1) can be regarded as a quantitative version of the well-known "simplicity conjecture" on the zeros of $\zeta(s)$, and also, as a weak version of

$$
\sum_{0<\Im \rho_{n} \leq T} \frac{1}{\left|\zeta^{\prime}\left(\rho_{n}\right)\right|^{2 k}}=O\left(T(\log T)^{(k-1)^{2}}\right) \quad(k \in \mathbb{R} ; k>0)
$$

with $k=1 / 2$, which is conjectured independently by Gonek [6] and Hejhal [7].

Under the above assumptions, we give the analytic continuation of $\Phi_{2}\left(s_{1}, s_{2} ; 1, \tilde{\alpha}\right)$. For this aim, we first recall some properties of $1 / \zeta(s)$.

Using the functional equation of $\zeta(s)$ (see [22, $\S 2.1]$ ):

$$
\zeta(s)=2 \Gamma(1-s) \sin \frac{\pi s}{2}(2 \pi)^{s-1} \zeta(1-s)
$$

we can obtain

$$
\begin{aligned}
\zeta^{\prime}(-2 k) & =\frac{\pi}{2} \cos \left(\frac{-2 k \pi}{2}\right) 2 \Gamma(1+2 k)(2 \pi)^{-2 k-1} \zeta(1+2 k) \\
& =\frac{(-1)^{k}(2 k) !}{2(2 \pi)^{2 k}} \zeta(1+2 k) \neq 0 \quad(k \in \mathbb{N}) .
\end{aligned}
$$

Therefore all trivial zeros of $\zeta(s)$ produce simple poles of $1 / \zeta(s)$. Hence we obtain the following. 
Lemma 4.2. Under Assumption 4.1, all poles of $1 / \zeta(s)$ are simple and the residues are

$$
\begin{gathered}
\operatorname{Res}_{s=\rho_{n}} \frac{1}{\zeta(s)}=\frac{1}{\zeta^{\prime}\left(\rho_{n}\right)}, \\
\operatorname{Res}_{s=-2 k} \frac{1}{\zeta(s)}=\frac{1}{\zeta^{\prime}(-2 k)}=\frac{(-1)^{k} 2(2 \pi)^{2 k}}{(2 k) ! \zeta(1+2 k)} \quad(k \in \mathbb{N}) .
\end{gathered}
$$

Proof. By the above fact and Assumption 4.1, the simplicity of each zero is obvious. Therefore we have

$$
\begin{gathered}
\operatorname{Res}_{s=\rho_{n}} \frac{1}{\zeta(s)}=\lim _{s \rightarrow \rho_{n}} \frac{s-\rho_{n}}{\zeta(s)}=\frac{1}{\zeta^{\prime}\left(\rho_{n}\right)}, \\
\operatorname{Res}_{s=-2 k} \frac{1}{\zeta(s)}=\lim _{s \rightarrow-2 k} \frac{s+2 k}{\zeta(s)}=\frac{1}{\zeta^{\prime}(-2 k)} .
\end{gathered}
$$

Substituting (4.3) into the second equation, we complete the proof of Lemma 4.2.

Applying the Mellin-Barnes formula (1.4), we obtain

$$
\begin{aligned}
\Phi_{2}\left(s_{1}, s_{2} ; 1, \widetilde{\alpha}\right) & =\sum_{m, n=1}^{\infty} \frac{\widetilde{\alpha}(n)}{m^{s_{1}+s_{2}}} \frac{1}{2 \pi i \Gamma\left(s_{2}\right)} \int_{(c)} \Gamma\left(s_{2}+z\right) \Gamma(-z)\left(\frac{n}{m}\right)^{z} d z \\
& =\frac{1}{2 \pi i \Gamma\left(s_{2}\right)} \int_{(c)} \Gamma\left(s_{2}+z\right) \Gamma(-z) \Phi(-z ; \widetilde{\alpha}) \zeta\left(s_{1}+s_{2}+z\right) d z,
\end{aligned}
$$

where $\Re s_{2}>\max \{1, \delta\}, \Re\left(s_{1}+s_{2}\right)>\max \{2,1+\delta\}$ and $\max \left\{-\Re\left(s_{2}\right), 1-\Re\left(s_{1}+s_{2}\right)\right\}<$ $c<\min \{-1,-\delta\}<0$.

As in the proof of Theorem 2.1, we shift the path $(c)$ to $(N-\varepsilon)$, and apply Lemma 4.2 to $\Phi_{2}\left(s_{1}, s_{2} ; 1, \widetilde{\alpha}\right)$. Here we recall the definition of the Bernoulli numbers $\left\{B_{n}\right\}_{n \geq 0}$, which are given by

$$
\frac{t e^{t}}{e^{t}-1}=\sum_{n=0}^{\infty} B_{n} \frac{t^{n}}{n !} .
$$

Then we can obtain

$$
\begin{aligned}
& \Phi_{2}\left(s_{1}, s_{2} ; 1, \widetilde{\alpha}\right) \\
& =\frac{\Gamma\left(s_{2}-\delta\right) \Gamma(\delta)}{\Gamma\left(s_{2}\right)} \operatorname{Res}_{s=\delta}\left(\frac{\Phi(s ; \alpha)}{\zeta(s)}\right) \zeta\left(s_{1}+s_{2}-\delta\right) \\
& -2 \Phi(0 ; \alpha) \zeta\left(s_{1}+s_{2}\right)-\sum_{\substack{k=1 \\
k: \text { odd }}}^{N-1}\left(\begin{array}{c}
-s_{2} \\
k
\end{array}\right) \frac{(k+1) \Phi(-k ; \alpha)}{B_{k+1}} \zeta\left(s_{1}+s_{2}+k\right) \\
& +\sum_{\substack{k=1 \\
k: \text { even }}}^{N-1}\left[( \begin{array} { c } 
{ - s _ { 2 } } \\
{ k }
\end{array} ) \left\{\frac{(-1)^{k / 2} 2(2 \pi)^{k} \Phi(-k ; \alpha)}{\zeta(1+k)}\right.\right. \\
& \left.\times\left(b_{k} \zeta\left(s_{1}+s_{2}+k\right)-\frac{1}{k !} \zeta^{\prime}\left(s_{1}+s_{2}+k\right)\right)+c_{k}(\alpha) \zeta\left(s_{1}+s_{2}+k\right)\right\} \\
& +\frac{1}{\Gamma\left(s_{2}\right)} \sum_{n=1}^{\infty} \Gamma\left(s_{2}-\rho_{n}\right) \Gamma\left(\rho_{n}\right) \frac{\Phi\left(\rho_{n} ; \alpha\right)}{\zeta^{\prime}\left(\rho_{n}\right)} \zeta\left(s_{1}+s_{2}-\rho_{n}\right) \\
& +\frac{1}{2 \pi i \Gamma\left(s_{2}\right)} \int_{(N-\varepsilon)} \Gamma\left(s_{2}+z\right) \Gamma(-z) \Phi(-z ; \widetilde{\alpha}) \zeta\left(s_{1}+s_{2}+z\right) d z
\end{aligned}
$$


for $N \in \mathbb{N}$, where $\varepsilon$ is a small positive number, $b_{k}$ is defined by (2.2) and $c_{k}(\alpha)$ is the constant term of the Laurent series expansion of $\Phi(s ; \alpha) / \zeta(s)$ at $s=-k$ for any even positive integer $k$, namely

$$
c_{k}(\alpha)=\frac{1}{2 \pi i} \int_{|\xi+k|=\frac{1}{2}} \frac{\Phi(s ; \alpha)}{\zeta(\xi)} \frac{1}{\xi+k} d \xi \quad(k \in \mathbb{N} ; k: \text { even }) .
$$

The validity of shifting the path of the integration can be shown as in Section 2 . In fact, we can find an arbitrarily large $t_{1}>0$ for which

$$
\frac{1}{\zeta\left(\sigma+i t_{1}\right)}=O\left(\left|t_{1}\right|^{A}\right) \quad(A: \text { a positive constant })
$$

holds for $-1 \leq \sigma \leq 2$ (see [22, p.218, Theorem 9.7]), while we can easily see by the functional equation that $1 / \zeta(s)$ is of polynomial order in $|t|$ in the half-plane $\sigma \leq-1$ as $|t| \rightarrow \infty$. Using these facts and the condition (III) of the class $\mathcal{A}$, we find that the argument in Section 2 works again.

The second, the third and the fourth term on the right-hand side of (4.4) are meromorphic on the whole space $\mathbb{C}^{2}$. The last integral can be analytically continued to the region $\left\{\left(s_{1}, s_{2}\right) \in \mathbb{C}^{2} \mid \Re s_{2}>-N+\varepsilon, \Re\left(s_{1}+s_{2}\right)>1-N+\varepsilon\right\}$ by using the condition (III) and noting that in this region the poles of the integrand are not on the path of integration. It follows from (2.6), (2.7) and Assumptions 4.1 that the fifth term on the right-hand side of (4.4) is convergent absolutely for all $\left(s_{1}, s_{2}\right) \in \mathbb{C}^{2}$ except for its singularities $s_{2}=-j+\rho_{n}\left(j \in \mathbb{N}_{0}\right)$ and $s_{1}+s_{2}=1+\rho_{n}$. Finally we note that the first term is also meromorphic on $\mathbb{C}^{2}$ and particularly vanishes when $\Phi(s ; \alpha)$ has no pole or $\delta=1$ except for the case $s_{2}=1$.

The above arguments lead to the following theorem. The last assertion can be proved by the same way as in Theorem 2.2 .

Theorem 4.3. Let $\alpha \in \mathcal{A}$. Under Assumption 4.1. $\Phi_{2}\left(s_{1}, s_{2} ; 1, \widetilde{\alpha}\right)$ can be continued meromorphically to the whole space $\mathbb{C}^{2}$ by (4.4). The possible singularities of $\Phi_{2}\left(s_{1}, s_{2} ; 1, \widetilde{\alpha}\right)$ are located only on the subsets of $\mathbb{C}^{2}$ defined by one of the following equations:

$$
\begin{aligned}
& s_{1}+s_{2}=1-k \quad\left(k \in \mathbb{N}_{0}\right), \\
& s_{2}=-k \quad(k \in \mathbb{N}, k \geq 2), \\
& s_{2}=-l+\rho_{n} \quad\left(l \in \mathbb{N}_{0}, n \in \mathbb{N}\right), \\
& s_{1}+s_{2}=1+\rho_{n} \quad(n \in \mathbb{N}), \\
& s_{2}=-l+\delta \quad\left(l \in \mathbb{N}_{0}\right), \\
& s_{1}+s_{2}=1+\delta,
\end{aligned}
$$

where the last two equations are omitted when $\Phi(s ; \alpha)$ has no pole or $\delta=1$. All of them are "true" singularities.

\section{Reverse values of $\Phi_{2}\left(s_{1}, s_{2} ; 1, \Lambda\right)$}

As stated in Remark 2.3. Akiyama, Egami and Tanigawa [1] observed that the value of multiple zeta function (1.3) at any point $\left(-m_{1}, \ldots,-m_{r}\right)\left(m_{1}, \ldots, m_{r} \in \mathbb{N}_{0}\right)$ on the sets of singularities is what is called a point of indeterminacy, whose "value" can be understood only as a limit value which depends on a limiting process. They considered

$$
\lim _{s_{1} \rightarrow-m_{1}} \lim _{s_{2} \rightarrow-m_{2}} \cdots \lim _{s_{r} \rightarrow-m_{r}} \zeta_{r}\left(s_{1}, s_{2}, \ldots, s_{r}\right),
$$


which they called the regular value. Akiyama and Tanigawa 22 further defined two different types of limit values at $\left(-m_{1}, \ldots,-m_{r}\right)$ by

$$
\begin{aligned}
& \lim _{s_{r} \rightarrow-m_{r}} \ldots \lim _{s_{2} \rightarrow-m_{2}} \lim _{s_{1} \rightarrow-m_{1}} \zeta_{r}\left(s_{1}, s_{2}, \ldots, s_{r}\right), \\
& \lim _{\varepsilon \rightarrow 0} \zeta_{r}\left(-m_{1}+\varepsilon,-m_{2}+\varepsilon, \ldots,-m_{r}+\varepsilon\right)
\end{aligned}
$$

which are called the reverse value and the central value, respectively (for generalizations, see also Sasaki [19] [20, Komori [12, Onozuka [18, and Onozuka, Wakabayashi and the first-named author [16]).

Checking the right-hand sides of (2.3) and (4.4), we can see that the same situation as above occurs for $\Phi_{2}\left(s_{1}, s_{2} ; 1, \Lambda\right)$ and for $\Phi_{2}\left(s_{1}, s_{2} ; 1, \widetilde{\alpha}\right)$. We aim to consider reverse values of them in this and the next section, because reverse values seem to be more interesting than regular values in the present situation (see Remark 5.5).

Here we define the reverse value of $\Phi_{2}\left(s_{1}, s_{2} ; 1, \Lambda\right)$ at $\left(u_{1}, u_{2}\right)$ on each singular set determined by

$$
\Phi_{2}^{\operatorname{Rev}}\left(u_{1}, u_{2} ; 1, \Lambda\right)=\lim _{s_{2} \rightarrow u_{2}} \lim _{s_{1} \rightarrow u_{1}} \Phi_{2}\left(s_{1}, s_{2} ; 1, \Lambda\right) .
$$

We give several examples of explicit formulas for reverse values by use of $\lim _{s \rightarrow-k} 1 / \Gamma(s)=$ 0 for $k \in \mathbb{N}_{0}$. Let $(s)_{k}:=s(s+1)(s+2) \cdots(s+k-1)$.

Proposition 5.1. Let $m, n \in \mathbb{N}_{0}$ with $2 \mid(m+n)$ and assume $m \geq 1$ when $n \geq 2$. Then

$$
\begin{gathered}
\Phi_{2}^{\operatorname{Rev}}(-m,-n ; 1, \Lambda) \\
=\frac{B_{m+n+2}}{(n+1)(m+n+2)}+(\log 2 \pi) \frac{B_{m+n+1}}{m+n+1} \\
-\sum_{\substack{k=1 \\
k: \text { odd }}}^{n}\left(\begin{array}{l}
n \\
k
\end{array}\right) \frac{k+1}{B_{k+1}} \zeta^{\prime}(-k) \frac{B_{m+n-k+1}}{m+n-k+1} \\
+\sum_{\substack{k=1 \\
k: \text { even }}}^{n}\left(\begin{array}{l}
n \\
k
\end{array}\right) \frac{B_{m+n-k+1}}{m+n-k+1}\left(-a_{k}+k ! b_{k}\right) \\
\quad-\frac{(-1)^{m} m ! n !}{(m+n+1) !} M(-m-n-1) .
\end{gathered}
$$

Proof. We write (2.3) as

$$
\begin{aligned}
& \Phi_{2}\left(s_{1}, s_{2} ; 1, \Lambda\right) \\
& =\frac{\zeta\left(s_{1}+s_{2}-1\right)}{s_{2}-1}-(\log 2 \pi) \zeta\left(s_{1}+s_{2}\right)+\sum_{o}-\sum_{e}-\sum_{\rho}+I,
\end{aligned}
$$

say. We let $\lim _{s_{2} \rightarrow-n} \lim _{s_{1} \rightarrow-m}$ on the both sides of (5.1) under $N>m+n+1$. Then we see that $\sum_{\rho} \rightarrow 0$ and $I \rightarrow 0$ because $\lim _{s_{2} \rightarrow-n}\left|\Gamma\left(s_{2}\right)\right| \rightarrow \infty$. Also, applying the well-known fact

(see [22, § 2.4]), we obtain

$$
\zeta(1-j)=-\frac{B_{j}}{j} \quad(j \in \mathbb{N})
$$

$$
\begin{aligned}
\sum_{o} \rightarrow & -\sum_{\substack{k=1 \\
k: \text { odd }}}^{n}\left(\begin{array}{l}
n \\
k
\end{array}\right) \frac{k+1}{B_{k+1}} \zeta^{\prime}(-k) \frac{B_{m+n-k+1}}{m+n-k+1} \\
& -\frac{(-1)^{m} m ! n !}{(m+n+1) !} M(-m-n-1)
\end{aligned}
$$


for $n \geq 1$, and

$$
\begin{aligned}
&-\sum_{e} \rightarrow \sum_{\substack{k=1 \\
k: \text { even }}}^{n} {\left[\left(\begin{array}{l}
n \\
k
\end{array}\right)\left\{\frac{B_{m+n-k+1}}{m+n-k+1}\left(-a_{k}+k ! b_{k}\right)-\zeta^{\prime}(-m-n+k)\right\}\right.} \\
&\left.+\left(\begin{array}{l}
n \\
k
\end{array}\right) \zeta^{\prime}(-m-n+k)\right]
\end{aligned}
$$

for $n \geq 2$ and $m \geq 1$. Here, in the calculations of $\sum_{o}$, the pole of $\zeta\left(s_{1}+s_{2}+k\right)$ for $k=m+n+1$ and $s_{1}=-m$ at $s_{2}=-n$ is cancelled with the zero of the binomial coefficient, namely,

$$
\begin{aligned}
& \left(\begin{array}{c}
-s_{2} \\
m+n+1
\end{array}\right) \zeta\left(s_{2}+n+1\right) \\
& =\frac{-s_{2}\left(-s_{2}-1\right) \cdots\left(-s_{2}-n+1\right)\left(-s_{2}-n\right)\left(-s_{2}-n-1\right) \cdots\left(-s_{2}-m-n\right)}{(m+n+1) !} \\
& \times\left(\frac{1}{s_{2}+n}+\cdots\right),
\end{aligned}
$$

and so, letting $s_{2} \rightarrow-n$, we have

$$
\begin{aligned}
\left(\begin{array}{c}
-s_{2} \\
m+n+1
\end{array}\right) \zeta\left(s_{2}+n+1\right) \rightarrow- & \frac{n(n-1) \cdots 1 \cdot(-1)(-2) \cdots(-m)}{(m+n+1) !} \\
& =\frac{(-1)^{m-1} m ! n !}{(m+n+1) !},
\end{aligned}
$$

because $m+n$ is even. Also in the calculations of $\sum_{e}$, we use (2.17), we have

$$
\lim _{s_{2} \rightarrow-n}-\frac{1}{k !} \frac{\Gamma^{\prime}\left(s_{2}+k\right)}{\Gamma\left(s_{2}\right)} \zeta\left(-m+s_{2}+k\right)=\left(\begin{array}{l}
n \\
k
\end{array}\right) \zeta^{\prime}(-m-n+k)
$$

for $n \geq 2$ and $m \geq 1$, since $\zeta(-m-n+k)=0$. Thus we complete the proof of Proposition 5.1.

Remark 5.2. As stated above, Proposition 5.1 holds for $m, n \in \mathbb{N}_{0}$ with $2 \mid m+n$. If $m+n$ is odd with $n \geq 2$, then $\Phi_{2}\left(s_{1}, s_{2} ; 1, \Lambda\right)$ is not convergent as $s_{1} \rightarrow-m$ and $s_{2} \rightarrow-n$. In fact, in this case, the right-hand side of (2.3) is not convergent because of the term $\Gamma^{\prime}\left(s_{2}+k\right) / \Gamma\left(s_{2}\right)$ for any even $k(\leq n)$. We emphasize that for the case $m+n$ is even, the pole of $\Gamma^{\prime}\left(s_{2}+k\right) / \Gamma\left(s_{2}\right)$ at $s_{2}=-n$ and the zero of $\zeta\left(s_{2}-m+k\right)$ at $s_{2}=-n$ are cancelled and this determines finite values.

Example 5.3. From Proposition 5.1, we obtain

$$
\begin{aligned}
& \Phi_{2}^{\mathrm{Rev}}(0,0 ; 1, \Lambda)=\frac{1}{2} \log 2 \pi+\frac{1}{12}-12 \zeta^{\prime}(-1), \\
& \Phi_{2}^{\mathrm{Rev}}(-1,0 ; 1, \Lambda)=\frac{1}{12} \log 2 \pi-\frac{3}{4}-\frac{\zeta^{\prime \prime}(-2)}{4 \zeta^{\prime}(-2)}+\frac{\gamma}{2}, \\
& \Phi_{2}^{\mathrm{Rev}}(-1,-1 ; 1, \Lambda)=-\frac{1}{240}-\zeta^{\prime}(-1)-20 \zeta^{\prime}(-3) .
\end{aligned}
$$

Proposition 5.4. For $l=0,1$ and $n \in \mathbb{N}$,

$$
\begin{aligned}
\Phi_{2}^{\mathrm{Rev}}\left(1+l+\rho_{n},-l ; 1, \Lambda\right) & =-(\log 2 \pi) \zeta\left(1+\rho_{n}\right)+\frac{\pi l \operatorname{lord}\left(\rho_{n}\right)}{\left(\rho_{n}\right)_{l+1} \sin \left(\pi \rho_{n}\right)} \\
& +\sum_{\substack{k=1 \\
k \text { :odd }}}^{l}\left(\begin{array}{l}
l \\
k
\end{array}\right) M(-k) \zeta\left(1+k+\rho_{n}\right) .
\end{aligned}
$$


Proof. We take the limit $s_{1} \rightarrow 1+l+\rho_{n}$ and then $s_{2} \rightarrow-l$ in (5.1) with $N=2$. Then $I$ vanishes and we obtain

$$
\begin{aligned}
\sum_{\rho} & =(-1)^{l} l \operatorname{lord}\left(\rho_{n}\right) \Gamma\left(-l-\rho_{n}\right) \Gamma\left(\rho_{n}\right) \\
& =-\frac{l ! \operatorname{ord}\left(\rho_{n}\right)}{\left(\rho_{n}\right)_{l+1}} \Gamma\left(1-\rho_{n}\right) \Gamma\left(\rho_{n}\right) \\
& =-\frac{\pi l \operatorname{ord}\left(\rho_{n}\right)}{\left(\rho_{n}\right)_{l+1} \sin \left(\pi \rho_{n}\right)}
\end{aligned}
$$

by the known formula $\Gamma(s) \Gamma(1-s)=\pi / \sin (\pi s)$.

Remark 5.5. We can also compute the regular values of $\Phi_{2}\left(s_{1}, s_{2} ; 1, \Lambda\right)$ at $\left(s_{1}, s_{2}\right)=$ $\left(u_{1}, u_{2}\right)$ on singular sets defined by

$$
\Phi_{2}^{\mathrm{Reg}}\left(u_{1}, u_{2} ; 1, \Lambda\right)=\lim _{s_{1} \rightarrow u_{1}} \lim _{s_{2} \rightarrow u_{2}} \Phi_{2}\left(s_{1}, s_{2} ; 1, \Lambda\right) .
$$

However it is almost trivial. In fact, if we consider this limit in (5.1) for $\left(u_{1}, u_{2}\right)=$ $(-m,-n)$ and $\left(k+1+\rho_{l},-k\right)\left(m, n, k \in \mathbb{N}_{0}, l \in \mathbb{N}\right)$, then the fifth and the sixth terms simply vanish, and the values of other terms can be computed just by substituting the limit values except for the cases which are not convergent as noted in Remark 5.2.

\section{Reverse values of $\Phi_{2}\left(s_{1}, s_{2} ; 1, \mu\right)$}

As stated in (3.1), we have

$$
\frac{1}{\zeta(s)}=\sum_{n=1}^{\infty} \frac{\mu(n)}{n^{s}} \quad(\Re s>1) .
$$

Hence, when $\Phi(s ; \alpha)=1$ in (1.8), we see that $\widetilde{\alpha}=\mu$ in this case. Note that $\Phi(s ; \alpha)=1$ has no pole.

Similarly, it is also known that

$$
\begin{aligned}
& \frac{\zeta(s-1)}{\zeta(s)}=\sum_{n=1}^{\infty} \frac{\phi(n)}{n^{s}} \quad(\Re s>2), \\
& \frac{\zeta(2 s)}{\zeta(s)}=\sum_{n=1}^{\infty} \frac{\lambda(n)}{n^{s}} \quad(\Re s>1),
\end{aligned}
$$

where $\phi$ is the Euler totient function and $\lambda$ is defined by $\lambda(n)=(-1)^{r}$, where $r$ is the number of prime factors with multiplicity (see $[22, \S 1.2]$ ). Therefore we can also apply Theorem 4.3 to these cases.

Here we consider the case $\Phi(s ; \alpha)=1$, namely $\widetilde{\alpha}=\mu$ and $c_{k}=c_{k}(\alpha)$ defined by (4.5). We can see that

$$
c_{k}=\lim _{s \rightarrow-k} \frac{d}{d s} \frac{s+k}{\zeta(s)}=-\frac{\zeta^{\prime \prime}(-k)}{2\left(\zeta^{\prime}(-k)\right)^{2}} \quad(k \text { :even }) .
$$

From (4.4) it follows that

$$
\begin{aligned}
& \Phi_{2}\left(s_{1}, s_{2} ; 1, \mu\right) \\
& =-2 \zeta\left(s_{1}+s_{2}\right)-\sum_{\substack{k=1 \\
k: \text { odd }}}^{N-1}\left(\begin{array}{c}
-s_{2} \\
k
\end{array}\right) \frac{(k+1) \zeta\left(s_{1}+s_{2}+k\right)}{B_{k+1}} \\
& +\sum_{\substack{k=1 \\
k: \text { even }}}^{N-1}\left[( \begin{array} { c } 
{ - s _ { 2 } } \\
{ k }
\end{array} ) \left\{\frac{(-1)^{k / 2} 2(2 \pi)^{k}}{\zeta(1+k)}\left(b_{k} \zeta\left(s_{1}+s_{2}+k\right)-\frac{\zeta^{\prime}\left(s_{1}+s_{2}+k\right)}{k !}\right)\right.\right.
\end{aligned}
$$




$$
\begin{aligned}
& \left.\left.+c_{k} \zeta\left(s_{1}+s_{2}+k\right)\right\}-\frac{\Gamma^{\prime}\left(s_{2}+k\right)}{\Gamma\left(s_{2}\right)} \frac{(-1)^{k / 2} 2(2 \pi)^{k}}{(k !)^{2} \zeta(1+k)} \zeta\left(s_{1}+s_{2}+k\right)\right] \\
+ & \frac{1}{\Gamma\left(s_{2}\right)} \sum_{n=1}^{\infty} \Gamma\left(s_{2}-\rho_{n}\right) \Gamma\left(\rho_{n}\right) \frac{\zeta\left(s_{1}+s_{2}-\rho_{n}\right)}{\zeta^{\prime}\left(\rho_{n}\right)} \\
+ & \frac{1}{2 \pi i \Gamma\left(s_{2}\right)} \int_{(N-\varepsilon)} \Gamma\left(s_{2}+z\right) \Gamma(-z) \frac{\zeta\left(s_{1}+s_{2}+z\right)}{\zeta(-z)} d z
\end{aligned}
$$

for $N \in \mathbb{N}$, where $\varepsilon$ is a small positive number, $b_{k}$ and $c_{k}$ are defined by (2.2) and (6.1). Therefore we can calculate the reverse value of $\Phi_{2}\left(s_{1}, s_{2} ; 1, \mu\right)$ at $\left(u_{1}, u_{2}\right)$ on singular sets determined by (4.7):

$$
\Phi_{2}^{\operatorname{Rev}}\left(u_{1}, u_{2} ; 1, \mu\right)=\lim _{s_{2} \rightarrow u_{2}} \lim _{s_{1} \rightarrow u_{1}} \Phi_{2}\left(s_{1}, s_{2} ; 1, \mu\right),
$$

using the same method as in the proofs of Propositions 5.1 and 5.4. As noted above, since $\Phi(s ; \alpha)=1$ has no pole, (4.7) implies

$$
\begin{aligned}
& s_{2}=-k \quad(k \in \mathbb{N}, k \geq 2) \\
& s_{1}+s_{2}=1-k \quad\left(k \in \mathbb{N}_{0}\right), \\
& s_{2}=-l+\rho_{n} \quad\left(l \in \mathbb{N}_{0}, n \in \mathbb{N}\right), \\
& s_{1}+s_{2}=1+\rho_{n} \quad(n \in \mathbb{N}) .
\end{aligned}
$$

For example, we can obtain the following from (6.2).

Example 6.1. Under Assumption 4.1,

$$
\begin{aligned}
& \Phi_{2}^{\operatorname{Rev}}(0,0 ; 1, \mu)=13, \\
& \Phi_{2}^{\operatorname{Rev}}(-1,0 ; 1, \mu)=\frac{1}{6}-\frac{2 \pi^{2}}{\zeta(3)}\left(\frac{3}{2}-\gamma\right)-\frac{\zeta^{\prime \prime}(-2)}{4 \zeta^{\prime}(-2)^{2}}, \\
& \Phi_{2}^{\operatorname{Rev}}\left(1+\rho_{n}, 0 ; 1, \mu\right)=-2 \zeta\left(1+\rho_{n}\right)-\frac{\pi}{\rho_{n} \zeta^{\prime}\left(\rho_{n}\right) \sin \left(\pi \rho_{n}\right)}, \\
& \Phi_{2}^{\operatorname{Rev}}\left(2+\rho_{n},-1 ; 1, \mu\right) \\
& \quad=-2 \zeta\left(1+\rho_{n}\right)-\frac{\pi}{\rho_{n}\left(\rho_{n}+1\right) \zeta^{\prime}\left(\rho_{n}\right) \sin \left(\pi \rho_{n}\right)}-12 \zeta\left(2+\rho_{n}\right) .
\end{aligned}
$$

\section{ACKNOWLEDGMENTS.}

The authors are sincerely grateful to Professors Hirotaka Akatsuka, Hideaki Ishikawa and Masatoshi Suzuki for their useful advice.

\section{REFERENCES}

[1] S. Akiyama, S. Egami and Y. Tanigawa, Analytic continuation of multiple zeta functions and their values at non-positive integers, Acta Arith. 98 (2001), 107-116.

[2] S. Akiyama, Y. TAnigawa, Multiple zeta values at non-positive integers, Ramanujan J. 5 (2001), $327-351$.

[3] R. DE LA BREtÈche, Estimation de sommes multiples de fonctions arithmétiques, Compositio Math. 128 (2001), 261-298.

[4] Y. Choie And K. Matsumoto, Functional equations for double series of Euler type with coefficients, Adv. Math. 292 (2016), 529-557.

[5] S. Egami And K. Matsumoto, Convolutions of the von Mangoldt function and related Dirichlet series, in Number theory, Sailing on the Sea of Number Theory, S. Kanemitsu and J.-Y. Liu (eds.), Proc. 4th China-Japan Seminar, Ser. Number Theory Appl. 2, World Sci. Publ. Hackensack, NJ, 2007, pp.1-23.

[6] S. M. GoneK, On negative moments of the Riemann zeta-function, Mathematika 36 (1989), 71-88. 
[7] D. Hejhal, On the distribution of $\log \left|\zeta^{\prime}\left(\frac{1}{2}+i t\right)\right|$, in Number Theory, Trace Formula and Discrete Groups, K. E. Aubert, E. Bombieri and D. Goldfeld (eds.), Academic press, San Diego, 1989, pp.343-370.

[8] A. E. Ingham, The Distribution of Prime Numbers, Cambridge Univ. Press, 1932.

[9] A. Ivić, The Riemann Zeta-Function. Theory and Applications, Dover Publications, Inc., Mineola, NY, 2003.

[10] M. Katsurada, An application of Mellin-Barnes' type integrals to the mean square of Lerch zetafunctions, Collect Math. 48 (1997), 137-153.

[11] M. Katsurada, An application of Mellin-Barnes type of integrals to the mean square of $L$ functions, Liet. Mat. Rink. 38 (1998), 98-112; Lithuanian Math. J. 38 (1998), 77-88.

[12] Y. Komori, An integral representation of multiple Hurwitz-Lerch zeta functions and generalized multiple Bernoulli numbers, Quart. J. Math. (Oxford) 61 (2010), 437-496.

[13] K. Matsumoto, On the analytic continuation of various multiple zeta-functions, in Number Theory for the Millennium II, M. A. Bennett et al. (eds.,), A K Peters, 2002, pp.417-440.

[14] K. Matsumoto, Asymptotic expansions of double zeta-functions of Barnes, of Shintani, and Eisenstein series, Nagoya Math. J. 172 (2003), 59-102.

[15] K. Matsumoto, The analytic continuation and the asymptotic behaviour of certain multiple zetafunctions I, J. Number Theory 101 (2003), 223-243.

[16] K. Matsumoto, T. Onozuka And I. WakABayashi, Laurent series expansions of multiple zetafunctions of Euler-Zagier type at integer points, preprint, arXiv:1601.05918

[17] K. Matsumoto and Y. Tanigawa, The analytic continuation and the order estimate of multiple Dirichlet series, J. Theor. Nombres Bordeaux 15 (2003), 267-274.

[18] T. OnOzUKA, Analytic continuation of multiple zeta-functions and the asymptotic behavior at non-positive integers, Funct. Approx. Comment. Math. 49 (2013), 331-348.

[19] Y. SASAKI, Multiple zeta values for coordinatewise limits at non-positive integers, Acta Arith. 136 (2009), 299-317.

[20] Y. SASAKI, Some formulas of multiple zeta values for coordinate-wise limits at non-positive integers, in New Directions in Value-Distribution Theory of Zeta and L-Functions, R. \& J. Steuding (eds.), Shaker, 2009, pp.317-325.

[21] E. M. Stein and R. Shakarchi, Complex Analysis, Princeton Lectures in Analysis, 2, Princeton University Press, Princeton, NJ, 2003.

[22] E. C. Titchmarsh, Revised by D. R. Heath-Brown, The Theory of the Riemann Zeta-function, Second edition, The Clarendon Press, Oxford University Press, New York, 1986.

[23] E. T. Whittaker and G. N. Watson, A Course of Modern Analysis, Reprint of the fourth (1927) edition. Cambridge Mathematical Library, Cambridge University Press, Cambridge.

[24] J. Zнао, Analytic continuation of multiple zeta functions, Proc. Amer. Math. Soc. 128 (2000), $1275-1283$.

K. Matsumoto, Graduate School of Mathematics,, Nagoya University,, Chikusa-ku, NAGOYA 464-8602, JAPAN

E-mail address: kohjimat@math.nagoya-u.ac.jp

A. Nawashiro, Dai Nippon Printing Co., Ltd.,, 1-1-1, Ichigaya-Kagacho,, Shinjuku-Ku, TOKYO, 162-8001, JAPAN

E-mail address: nawashiro-a@mail.dnp.co.jp

H. Tsumura, Department of Mathematical Sciences,, Tokyo Metropolitan University,, 1-1, Minami-Ohsawa, Hachiouji, Tokyo, 192-0397, Japan

E-mail address: tsumura@tmu.ac.jp 